\title{
Top-down modulation of the perception of other people in schizophrenia and autism
}

\author{
Jennifer Cook ${ }^{1 *}$, Guillaume Barbalat ${ }^{1}$ and Sarah-Jayne Blakemore ${ }^{2}$ \\ Department of Psychiatry, University of Cambridge, London, UK \\ 2 Institute of Cognitive Neuroscience, University College London, London, UK
}

\section{Edited by:}

Chris Frith, Wellcome Trust Centre

for Neuroimaging at University

College London, UK

Reviewed by:

Antonia Hamilton, University

of Nottingham, UK

Valerian Chambon, Institut National

de la Santé et de la Recherche

Médical, France

\section{*Correspondence:}

Jennifer Cook, Department of Psychiatry, University of Cambridge, Herchel Smith Building, Cambridge Biomedical Campus, Cambridge,

CB1 7AJ, UK

e-mail:jc746@cam.ac.uk
Accurately and efficiently perceiving social cues such as body movements and facial expressions is important in social interaction. Accurate social perception of this kind does not solely rely on "bottom-up" visual processing but is also subject to modulation by "top-down" signals. For example, if instructed to look for signs of happiness rather than fear, participants are more likely to categorize facial expressions as happy-this prior expectation biases subsequent perception. Top-down modulation is also important in our reactions to others. For example, top-down control over imitation plays an important role in the development of smooth and harmonious social interactions. This paper highlights the importance of top-down modulation in our perception of, and reactions to, others. We discuss evidence that top-down modulation of social perception and imitation is atypical in Autism Spectrum Conditions and in schizophrenia, and we consider the effect this may have on the development of social interactions for individuals with these developmental disorders.

Keywords: autism, schizophrenia, top-down, modulation, social perception, attention, expectation, priming

\section{TOP-DOWN MODULATION IS IMPORTANT IN SOCIAL INTERACTION}

It is important for social interaction that individuals have efficient and accurate mechanisms for social perception. Accurate social perception depends on a number of processes including "bottom-up" sensory processing and "top-down" modulation, which prioritizes the stimuli that are most relevant to our current activities and goals. This paper focuses on top-down processes involved in perception of and reactions to social stimuli.

Top-down control involves multiple processes, including most notably attention and expectation. For example, if asked to direct your attention to a particular face that is hidden amongst a crowd of faces, you would be more efficient at detecting its characteristics compared to those of the other faces in the crowd. We are also able to make predictions about the nature of an incoming stimulus based on our prior expectations. Suppose that you are at a pub with some friends; the general ambiance is warm and happy, you expect your peers to show positive facial expressions. However, you might anticipate encountering different facial expressions in another context; for instance, one is more likely to observe expressions of sorrow at a funeral. Contradictions of our expectations in either case will surprise us. In contrast, the ability to anticipate others' emotions facilitates our behavioral response to the sensory world by promoting efficient sensory processing of stimuli that are congruent with expectations.

Recent theoretical (Summerfield and Egner, 2009) and computational (Wyart et al., 2012) models of visual processing dissociate attention and expectation. Whereas expectation facilitates visual perception by increasing the prior likelihood that a subset of visual information will occur, attention reduces the computational burden by prioritizing processing of a particular subset of visual information on the basis of its behavioral significance (Summerfield and Egner, 2009). Recent explanations have described the difference between attention and expectation in the context of signal-to-noise detection where enhanced signal-tonoise precision can be a result of increased signal or reduced noise (Wyart et al., 2012). Cues predicting the relevant location (attentional cues) of a to-be-detected signal primarily increase signalto-noise precision by suppressing noise; whereas, cues predicting greater signal probability (expectation cues) increase precision by elevating signal (Wyart et al., 2012).

The top-down effects of attention and expectation do not operate only at the level of perception but can also modulate our actions. One example of this is the modulation of imitation by social context. Individuals imitate more when in a positive social context (Lakin and Chartrand, 2003) and simple social ostensive cues such as direct eye-gaze affect automatic imitation mechanisms: individuals are faster to perform actions that match, rather than deviate from, observed actions (Wang et al., 2011a).

Even pro-social, compared to non-social, priming that is subliminal results in significantly higher levels of automatic imitation (Leighton et al., 2010; Cook and Bird, 2011a,b). For example, Leighton et al. (2010) asked participants to rearrange five words such that they formed a grammatically correct sentence; these sentences either comprised positive social words (e.g., friend, team, assist) or anti-social words (e.g., rebel, obstinate, distrust). Despite reporting no awareness of the underlying theme, individuals who had rearranged the positive social words exhibited higher levels of automatic imitation than individuals who had rearranged the anti-social words. Such facilitation of imitation according to social context is likely a key component in the development of smooth and harmonious social interactions: being 
imitated increases rapport (Chartrand and Bargh, 1999), altruistic behavior (van Baaren et al., 2004) and trust (Bailenson and Yee, 2005).

\section{NEURAL MECHANISMS OF TOP-DOWN MODULATION OF SOCIAL PERCEPTION}

In recent years a number of studies have investigated the neural mechanisms that underpin the top-down modulation of social perception.

\section{ATTENTION}

The modulatory effects of attention are thought to proceed via feedback connections from frontal and parietal regions including the superior parietal lobule, intraparietal sulcus, frontal eye fields, and supplementary eye fields (Corbetta et al., 1993; Fink et al., 1997) to sensory processing areas (Cavada and GoldmanRakic, 1989; Ungerleider et al., 1989; see Figure 5 for example relating to auditory sensory processing). Such feedback connections are thought to play a role in amplifying activity in stimulus-specific neural regions (Kastner and Ungerleider, 2001; Pessoa et al., 2002). For instance, activity in motion specific visual cortex, and connectivity between early visual cortex (V2) and visual motion processing areas (hMT/V5), was enhanced when attending to visual motion compared to when not attending (Büchel et al., 1998). This enhanced connectivity was modulated by top-down signals from parietal and prefrontal cortex.

This explanatory framework, which states that top-down signals from parietal and frontal areas enhance stimulus processing in stimulus-specific cortex, extends to the processing of social stimuli. Attention to faces (compared with attention to a location that does not feature faces) is associated with increased activity in the fusiform face area (FFA; Vuilleumier et al., 2001; Bird et al., 2006) and the amygdala (Pessoa et al., 2002). Attention to faces is also associated with increased FFA to V1 connectivity (Bird et al., 2006).

\section{EXPECTATION}

Top-down signals relating to prior expectations, from frontal and parietal regions, enhance processing in stimulus-specific cortex. For instance, Summerfield et al. (2006) showed participants images of faces, houses, and cars. In each block participants were required to press a specific "target" button upon perceiving a particular stimulus type (e.g., face) and to press the "non-target" button for all other stimuli (e.g., cars and houses). It has previously been demonstrated that, in contrast to instructions such as "is the stimulus A (e.g., a face) or B (e.g., a car)?" instructions of the form "is the stimulus A or not?" involve the activation of a prior expectation (also referred to as an internal template; (Dayan et al., 1995; Dosher and Lu, 1999) against which all stimuli are compared (Summerfield and Koechlin, 2008). On each trial the participant therefore has a prior expectation for one stimulus type over the alternatives. In the paradigm employed by Summerfield and colleagues the prior expectation (that is, the stimulus-type to be detected) changed on a block-by-block basis. Analyses revealed enhanced activity in the ventro-medial prefrontal cortex (vMPFC) when the prior expectations matched the incoming sensory data (Summerfield and Koechlin, 2008).
Furthermore, connectivity analyses suggested a top-down influence of frontal cortex activity on face-responsive regions in the fusiform gyrus and the amygdala (Summerfield et al., 2006). Previous studies have suggested that vmPFC plays a role in confirming and reinforcing the validity of prior expectations (Daw et al., 2006; Hampton et al., 2006).

We recently investigated the influence of prior expectations on emotional facial expression discrimination. Prior expectations were first set by instructing participants to look out for faces with a particular "target" expression (fear, anger or happiness). Subsequently participants viewed a sequence of faces and responded with one button for the target expression and a different button for all other facial expressions. Detection responses were faster and more accurate for faces that matched prior expectations relative to non-matching faces. Furthermore, neuroimaging data showed that congruency, compared to incongruency, between prior expectation and incoming sensory data was associated with vmPFC activity (Barbalat et al., 2012a). In addition, there was greater functional connectivity between the vmPFC and the thalamus when an incoming angry face stimulus was congruent with the instruction, compared to when it was incongruent. The thalamus acts as an intermediary between the retina and emotion-processing areas (such as the amygdala) enabling rapid and preconscious processing of potentially threatening stimuli (Pessoa and Adolphs, 2010). Therefore it may be that when an individual is faced with a stimulus that matches prior expectation the vmPFC facilitates emotional responsiveness via top-down control of the thalamus.

\section{TOP-DOWN INFLUENCES ON ACTION OBSERVATION AND IMITATION}

In addition to their role in amplifying processing in stimulusspecific cortex, top-down signals may also modulate activity in action-related areas such as the mirror neuron system (MNS). Mirror neurons fire during both execution of an action and observation of that same action (di Pellegrino et al., 1992; Kraskov et al., 2009). Areas of the human brain with these response properties have been called the MNS (Iacoboni, 2009) and it has been suggested that the MNS comprises the neural correlate of imitation (Iacoboni, 2005, 2009). This hypothesis has been supported by findings that MNS areas are active during the imitation of actions (Iacoboni et al., 1999) and applying repetitive transcranial magnetic stimulation to disrupt activity in MNS areas results in reduced automatic imitation (Catmur et al., 2009) and higher error rates for effortful imitation (Heiser et al., 2003).

Although the MNS may automatically respond to observed actions, and likely supports imitation, we do not imitate every action we observe. Recent studies suggest that top-down signals from other, non-MNS, brain regions modulate MNS regions and hence control imitative responses. Following from observations that individuals with medial prefrontal cortex (mPFC) lesions exhibit heightened levels of imitation (Lhermitte, 1986; Brass et al., 2003), Brass and colleagues have used functional magnetic resonance imaging (fMRI) to show that inhibition, compared to execution, of imitative responses elicits activity in key nodes in the "social brain" network: the mPFC and temporo-parietal junction (TPJ) (Brass et al., 2001, 2003, 2005; Spengler et al., 2009, 
2010b). This association between control of imitation and social brain activity has been further supported in a series of studies by Hamilton and colleagues: direct eye contact increases automatic imitation of hand movements (Wang et al., 2011a); and this effect appears to be driven by the modulatory influence of mPFC activity on activity in action perception areas [posterior superior temporal sulcus (pSTS)], which subsequently influences activity in a key MNS region [inferior frontal gyrus (IFG)] (Wang et al., 2011b).

\section{ATYPICAL TOP-DOWN MODULATION IN AUTISM SPECTRUM CONDITIONS}

Autism spectrum conditions (ASC) are pervasive developmental disorders, characterized by a triad of impairments: verbal and non-verbal communication problems, difficulties with reciprocal social interactions, and unusual patterns of repetitive behavior (American Psychiatric Association, 1994). An accumulating body of evidence suggests atypical top-down modulation of early sensory processing in individuals with ASC (Frith, 2003). Bird et al. (2006) used fMRI to record brain activity from high functioning participants with ASC, and control participants, during the attentional modulation of face processing. Four images (two face images and two house images) were presented on each trial; images were arranged in a diamond shape with images from the same category in the same dimension (as in Figure 1). Before each trial, attention was directed to either the vertical or horizontal dimension; therefore, on each trial participants attended either to faces or to houses. For control participants, attention modulated activity in the FFA such that FFA activity was high when faces were attended and low when faces were not attended. Individuals with ASC did not demonstrate this same effect: FFA activity did not discriminate trials in which the face was and was not attended. Thus, this study demonstrated reduced attentional modulation of face processing in adults with ASC.

In addition to atypical effects of attention in ASC previous studies have demonstrated reduced effects of prior knowledge. For instance, for typical controls, viewing images of faces can improve subsequent identification of degraded versions of these faces; this effect is reduced in ASC (Loth et al., 2010). Similarly, Dichter and Belger (2008) demonstrated a lack of arousal mediated top-down modulation in ASC. Specifically they reported that when stimuli demanding cognitive control were preceded by highly arousing pictures, activation in the right middle frontal gyrus was elevated for control participants, compared to when stimuli were preceded by pictures that were low in arousal. In contrast, for individuals with ASC highly arousing pictures did not modulate right middle frontal gyrus activity, suggesting a lack of top-down modulation.

As discussed above, social actions such as imitation can be modulated by primed concepts. We recently demonstrated that the social modulation of imitation is diminished in individuals with ASC (Cook and Bird, 2011a). This study employed an adapted version of the paradigm used by Leighton et al. (2010) in which participants first completed a pro- or non-social priming task before completing a measure of automatic imitation. Whereas control participants primed with pro-social attitudes imitated significantly more than those primed with non-social

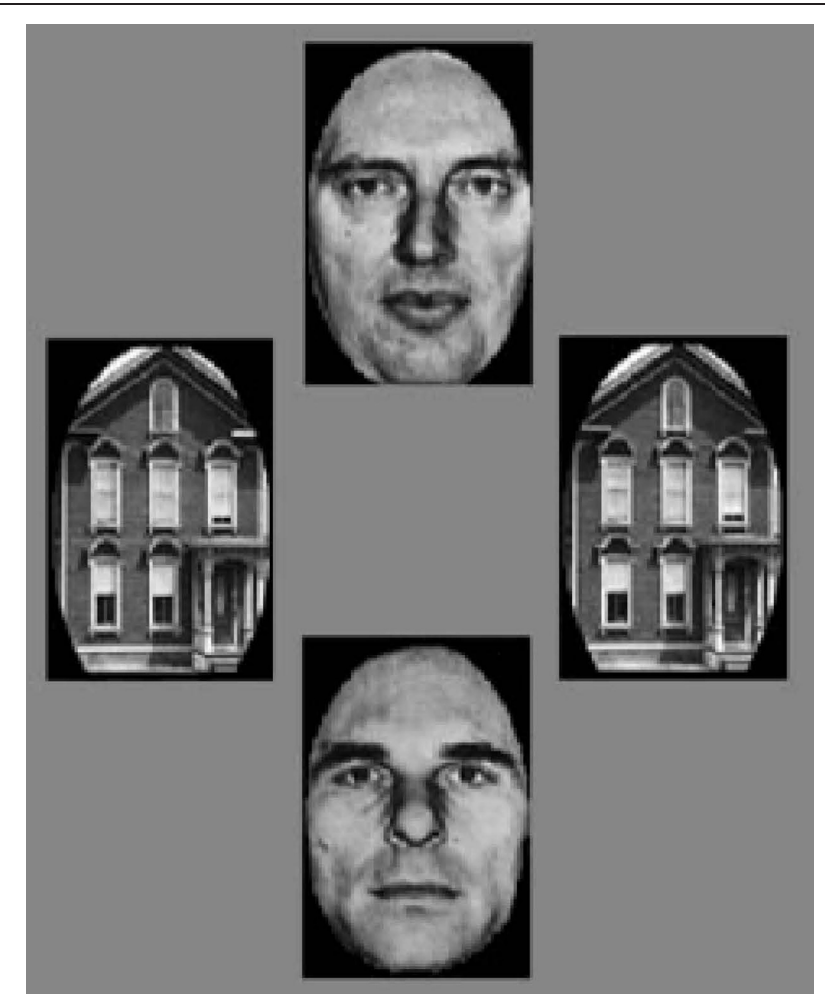

FIGURE 1 | Stimuli employed by Bird et al. (2006). Four images (two face images and two house images) were presented on each trial; images were arranged in a diamond shape with images from the same category (faces or houses) in the same dimension. For instance here faces are presented in the vertical dimension and houses in the horizontal dimension. Figure is reproduced, with permission, from Bird et al. (2006).

attitudes, this modulation of imitation was not seen for individuals with ASC. Participants with ASC primed with pro-social attitudes imitated to the same extent as those primed with non-social attitudes (Figure 2).

\section{NEURAL BASIS OF ATYPICAL TOP-DOWN MODULATION IN ASC}

Top-down modulation refers to the effects of signals from "control" regions, such as the PFC, on sensory processing. According to this definition there are at least three possible causes of atypical top-down modulation: atypical function of sensory regions; atypical function of control regions; and atypical connectivity between control and sensory regions. We discuss each of these in turn.

\section{SENSORY PROCESSING IN ASC}

A number of studies have suggested atypical basic sensory processing of social stimuli in ASC. Early imaging studies reported that individuals with ASC exhibit hypoactivation, relative to control participants, of the FFA when viewing faces, suggesting that abnormalities in this region may be related to atypical face processing in ASC (Critchley et al., 2000; Schultz et al., 2000; Pierce et al., 2001). A more recent study has shown that, when control participants and individuals with ASC are cued with a fixation cross to look at and attend to faces, FFA activity for individuals 


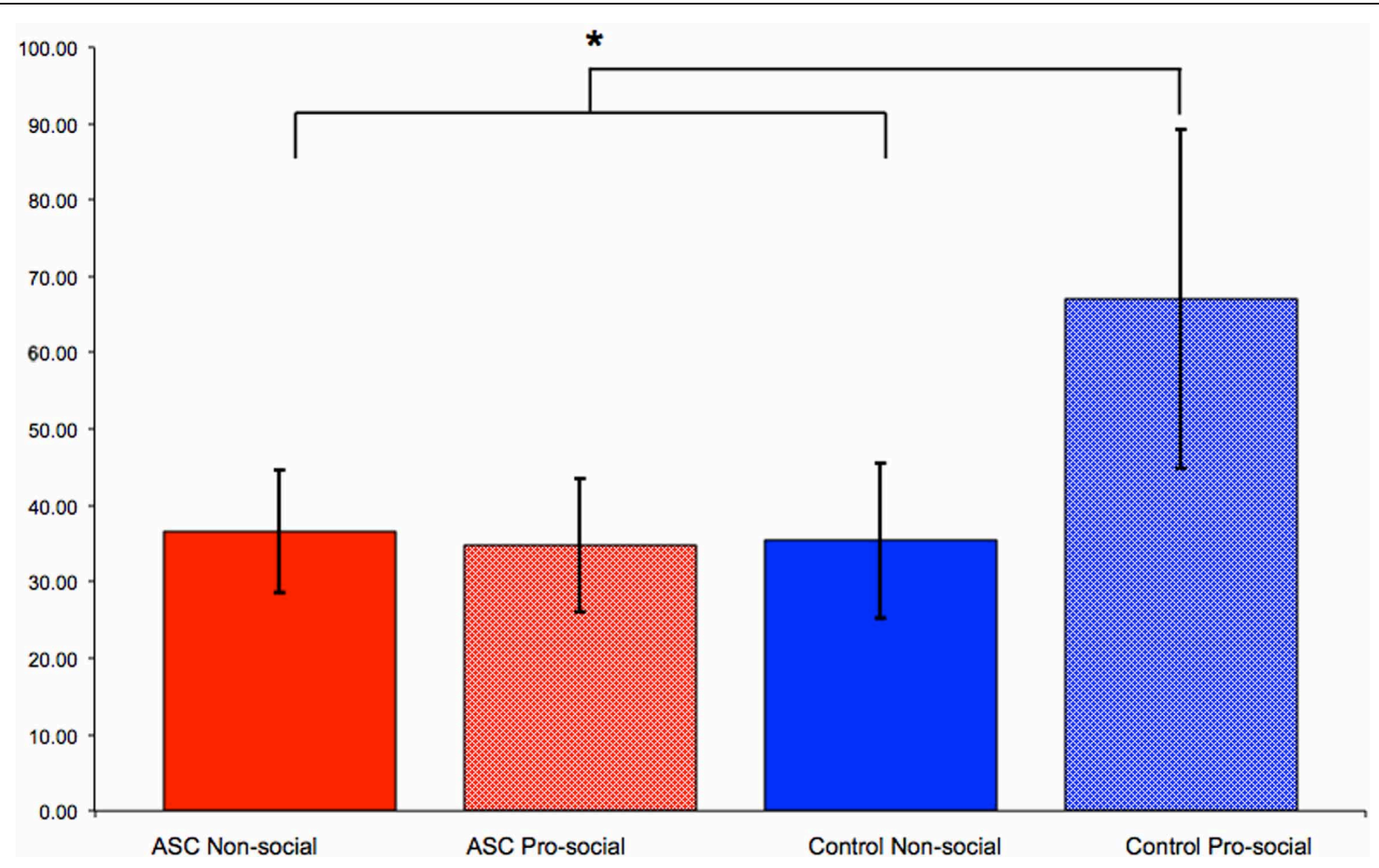

FIGURE 2 | Results from Cook and Bird (2011a). Control participants primed with pro-social attitudes imitated significantly more than those primed with non-social attitudes. However, this social modulation of imitation was not observed for individuals with ASC: those primed with pro-social attitudes imitated to the same extent as those primed with non-social attitudes. "*" indicates $p<0.05$. with ASC does not differ from that of age- and IQ- matched controls (Hadjikhani et al., 2004). A similar trend can be observed in the MNS literature whereby early studies suggested reduced MNS activation (Oberman et al., 2005; Dapretto et al., 2006) and corresponding imitation impairments (Avikainen et al., 2003; Rogers et al., 2003; McIntosh et al., 2006) in ASC. However, more recent imitation studies show that if individuals with ASC are forced to look at the relevant features of an action, thereby ensuring that they receive typical inputs about action kinematics, imitation is normalized (Bird et al., 2007; Press et al., 2010). Recent studies therefore suggest that when input to perception and action regions is controlled (e.g., by instructed direction of eye-gaze) individuals with ASC exhibit typical responses in sensory regions.

\section{FUNCTION OF CONTROL REGIONS IN ASC}

A number of studies have demonstrated that the mPFC and TPJ are key areas in the control of imitation (Lhermitte, 1986; Brass et al., 2001, 2005; Spengler et al., 2009, 2010b; Wang et al., 2011b). It has recently been shown that atypical mPFC activity during mentalising tasks is functionally associated with atypical control over imitation in ASC (Spengler et al., 2010a; see Figure 3). Spengler and colleagues' study consisted of three phases in which participants: (1) were scanned whilst watching animations that evoked mentalising (Castelli et al., 2000); (2) completed a behavioral measure of mentalising in which they had to infer the mental states of story characters (Happé et al., 1999); and (3) completed a behavioral measure of imitation-inhibition that required them to inhibit imitating a video of finger actions in order to make the required finger action. The ability to inhibit the tendency to imitate was associated with reduced behavioral mentalising scores and reduced social interaction scores on an ASC diagnostic instrument (the Autism Diagnostic Observation Schedule; Lord et al., 1989). Furthermore, mPFC and TPJ activity during the fMRI mentalising task was correlated with imitation-inhibition such that individuals with low mPFC activity exhibited poor imitation-inhibition. Thus, mentalising plays a role in imitation inhibition and atypical regulation of imitation in ASC may stem from aberrant mPFC activation.

\section{FUNCTIONAL CONNECTIVITY IN ASC}

Recent functional connectivity MRI (fcMRI) studies have suggested that functional connectivity between social brain regions such as the mPFC and MNS is atypical in ASC. Intrinsic fcMRI detects the temporal correlation between spatially discrete lowfrequency fluctuations of the BOLD signal. Shih et al. (2010) used fcMRI to investigate the intrinsic connectivity of brain areas associated with imitation and its control: the mPFC, IFG, inferior parietal lobe (IPL) and STS. FMRI data were collected while participants performed a non-imitative task (semantic decision/letter detection). The influence of PFC on MNS activity was atypical in ASC (Figure 4). In individuals with ASC under connectivity between frontal and posterior regions, during mentalising, has also been reported (Castelli et al., 2002; Kana et al., 2009). 


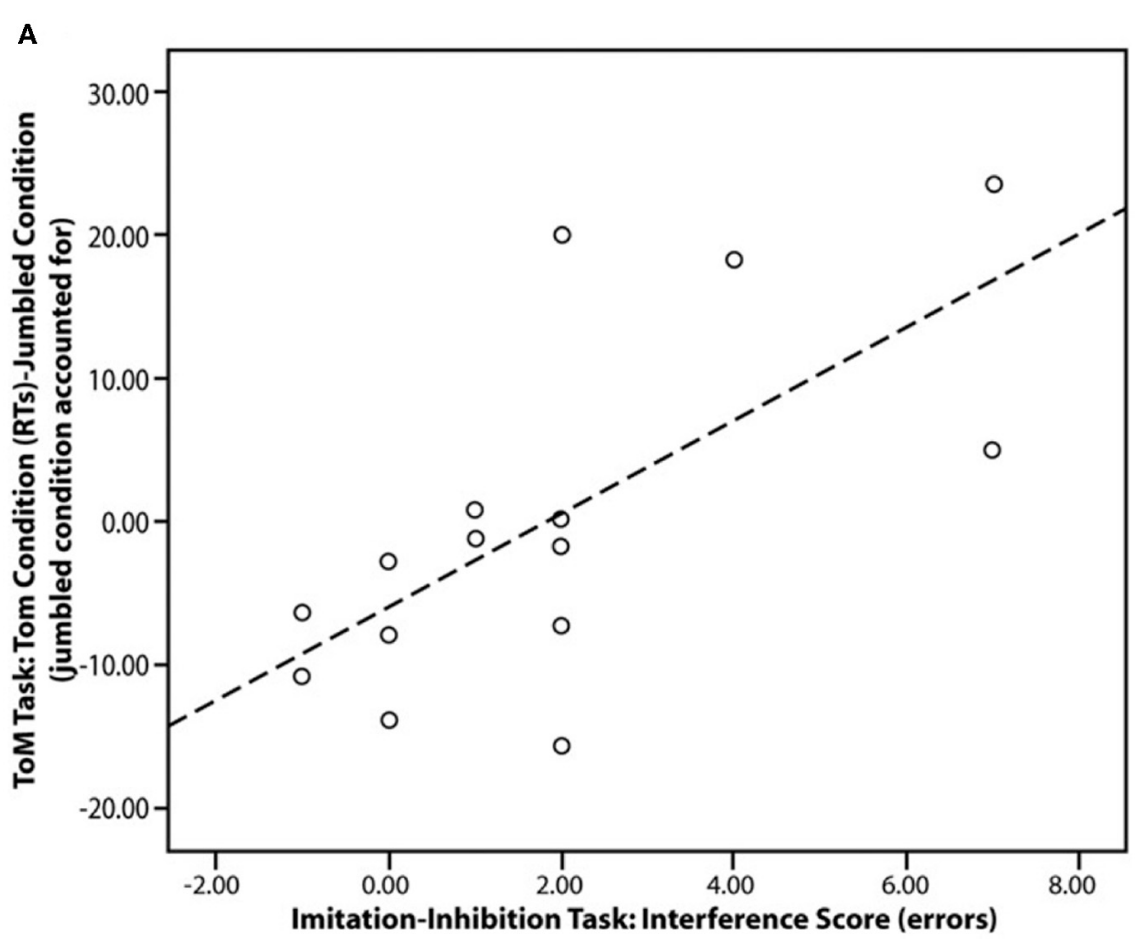

FIGURE 3 | Scatter plot showing the significant correlation in the ASC group between imitation-inhibition (interference score) and reaction times in the mentalising task (ToM, Theory of Mind condition). Figure is reproduced, with permission, from Spengler et al. (2010a).

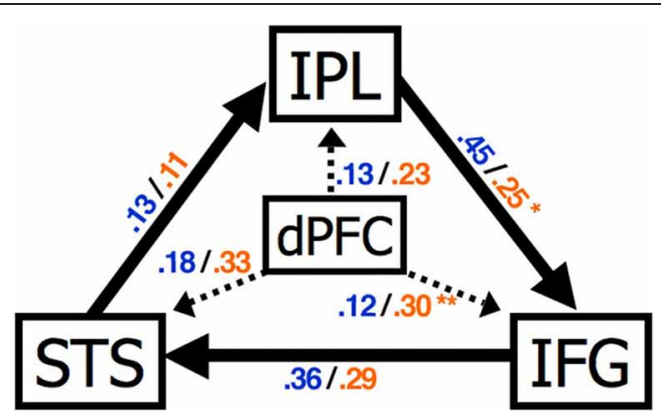

FIGURE 4 | Abnormal connectivity in the brain in ASC. Compared to control participants individuals with ASC showed a significantly increased effect of dPFC on IFG and reduced effect of IPL on IFG. STS, superior temporal sulcus; IPL, inferior parietal lobule; IFG, inferior frontal gyrus; $\mathrm{dPFC}$, dorsal prefrontal cortex. Figure is reproduced, with permission, from Shih et al. (2010).

\section{SUMMARY}

We suggest that atypical top-down modulation in ASC is due to a deficit in at least one of the following areas: sensory regions; control regions; and/or connectivity between control and sensory regions. Although not the focus of the current paper one may speculate that atypicalities in these areas could arise through both genetic and experiential avenues. Let us take top-down modulation of imitation as an example. As discussed above, top-down signals from frontal to MNS regions are the likely neural correlates of this phenomenon. Theoretically, atypical function of these brain regions or connectivity between the regions could be genetically predisposed or could be experientially acquired-if individuals with ASC are not motivated to participate in social situations (Chevallier et al., 2012), they will be less likely than controls to acquire links between pro-social contextual cues and elevated MNS activity. If these neural atypicalities are genetically predisposed they should be invariant to training. Future studies, which attempt to train top-down modulation in ASC, may shed light on the aetiological basis of this deficit.

\section{ATYPICAL TOP-DOWN MODULATION IN SCHIZOPHRENIA}

Schizophrenia is characterized by positive symptoms (e.g., hallucinations, delusions), negative symptoms (e.g., blunted affect, anhedonia), lack of motivation (e.g., avolition, social isolation), and cognitive impairments (e.g., working memory, attention). In the social domain, individuals with schizophrenia often demonstrate social awkwardness and difficulties in daily living (Penn et al., 1997). It has been argued that social cognition deficits represent a specific domain of impairment in this condition, independent from classical cognitive deficits (Penn et al., 1997) and that social impairments are highly resistant to medication (Penn et al., 2008). As such, social deficits represent an important domain of investigation in schizophrenia.

\section{TOP-DOWN INFLUENCE OF ATTENTION IN SCHIZOPHRENIA}

A number of studies have demonstrated reduced attentional modulation of non-social stimuli in schizophrenia. Individuals with schizophrenia exhibit impaired performance on oddball 
tasks, in which participants are required to respond to an infrequently presented target embedded in a stream of distractors [see Cornblatt and Keilp (1994) for review]. For control participants activity in PFC regions differentiates oddballs from nontarget stimuli (Kirino et al., 2000). Individuals with schizophrenia show abnormal frontal activations during such tasks (Kiehl and Liddle, 2001). Furthermore, individuals at high-risk of developing schizophrenia show significantly smaller differential frontal activations between oddballs and non-target stimuli, suggesting that prefrontal function begins to decline even before the onset of illness (Morey et al., 2005).

Dichter et al. (2010) recently used a modified version of a visual oddball fMRI task to investigate the influence of emotion on selective attention in schizophrenia. Participants were required to detect oddballs in a stream of distractors which included aversive emotional scenes, requiring participants to inhibit responses to the emotionally salient stimuli to achieve optimal task performance. Compared with controls, individuals with schizophrenia showed smaller differential frontal activations for oddballs and non-target stimuli. In addition, for oddballs relative to non-targets, controls deactivated limbic regions including the amygdala, whereas individuals with schizophrenia did not. Thus compared to individuals with schizophrenia, control participants were better able to inhibit their emotional reactions to aversive scenes. Dichter and colleagues also found that activation of frontal regions to the aversive stimuli was negatively correlated with avolition and anhedonia as measured by the Scale for the Assessment of Negative Symptoms (SANS, Andreasen, 1983). No correlations were found between BOLD response and positive symptoms as measured by the Scale for the Assessment of Positive Symptoms (SAPS, Andreasen, 1984). Suggesting that atypical frontal activations to aversive emotional stimuli may, in particular, be associated with the negative features of schizophrenia.

Although the work of Dichter and colleagues hints at atypical top-down attentional modulation in schizophrenia further work is necessary both to replicate this initial finding and to investigate more directly the influence of attention on the processing of social stimuli. Future work might employ a paradigm like that used by Bird et al. (2006) to investigate attentional modulation of face processing.

\section{PRIOR EXPECTATIONS IN SCHIZOPHRENIA}

Schizophrenia has been linked to an increased influence of prior expectations on sensory perception (Aleman et al., 2003). We recently compared the influence of prior expectations on facial expression discrimination in control participants and patients with schizophrenia (Barbalat et al., 2012b). Using the paradigm described above (Barbalat et al., 2012a), we found that, relative to controls, participants with schizophrenia were slower to identify a fearful face when instructed to look for an angry face and were less accurate to identify an angry face in a fearful context. Hence the incongruent prior expectation interfered more with the processing of incoming sensory data for individuals with schizophrenia than for controls. Such an increased influence of prior expectations in patients was not observed for happy faces, suggesting a specific over-weighting of prior expectations of negative emotions in schizophrenia. It has previously been suggested that over-reliance on prior expectations of negative emotions might specifically underlie delusions of persecution (Blackwood et al., 2001). In support of this, we found that patients with paranoid delusions were more biased by expectations of threat than patients who were not currently experiencing paranoid delusions. These results are in line with previous reports that a probabilistic reasoning impairment in schizophrenia is more prominent for salient stimuli such as threatening emotions (Blackwood et al., 2001).

A further example, of an atypical influence of prior expectations in schizophrenia, is illustrated in belief inflexibility (Woodward et al., 2008). Belief inflexibility is a thinking style in which patients show an unwillingness to modify their beliefs even when confronted with disconfirmatory evidence. In one demonstration of belief inflexibility participants were presented with an initial statement (e.g., "Heike is very thin") and asked to rate the probability that each of four possible explanations was true. These possible explanations ranged in the extent to which they were likely to be true [e.g., "Heike is homeless" (true), "Heike is a model" (lure), "Heike is suffering from an eating disorder" (lure), "Heike has lost her false teeth" (absurd)]. Following these initial ratings participants were presented with a second statement (e.g., "Heike has had a hard life") and asked if they would like to revise their original ratings. Finally participants were presented with a third statement (e.g., "Heike does not even have a home") and again asked if they would like to revise their ratings. For beliefs that were initially held strongly, patients with schizophrenia were less likely than control participants to revise their belief after the additional statements. It has been proposed that this maintenance of false beliefs in the face of disconfirmatory evidence may be related to patients giving too much weight to priors as compared to incoming sensory evidence (Moritz and Woodward, 2006; Woodward et al., 2006, 2008).

A growing body of evidence suggests atypical top-down influences of priming in schizophrenia. Ilankovic et al. (2011) asked participants, with and without paranoid schizophrenia, to listen to either self-produced or other-produced speech, which was either preceded by a photo of the participant or of the other speaker. On "valid" trials the participant viewed a photograph of the true speaker, whereas on "invalid" trials the photograph did not depict the speaker (e.g., the photo of the other was followed by self-produced speech). On each trial participants were required to judge whether speech was self-produced or other-produced. Individuals with paranoid schizophrenia made more errors in the invalid condition compared to control participants, suggesting that they were more susceptible to the top-down priming influence of the photograph. In the schizophrenia group delusion scores (as measured by the psychotic symptom rating scale PSYRATS; Haddock et al., 1999) were positively correlated with errors on invalid trials in which participants listened to their distorted voice preceded by the face of the other. Thus suggesting that an over-reliance on primes might be particularly strongly associated with delusions in schizophrenia.

Evidence from affective priming studies also suggests atypically strong priming effects in schizophrenia (Höschel and Irle, 2001; Suslow et al., 2003). Following subliminally presented negative facial expression primes individuals with schizophrenia were 
more likely than controls to judge neutral faces and objects as unpleasant. Similarly, following the viewing of negative scenes, compared to control participants, individuals with schizophrenia were more likely to rate faces as untrustworthy (Hooker et al., 2011). This body of evidence therefore suggests an abnormally strong influence of top-down negative primes on social stimulus processing.

\section{NEURAL BASIS OF ATYPICAL TOP-DOWN MODULATION IN SCHIZOPHRENIA}

Prominent theories of top-down cognitive biases in schizophrenia suggest abnormal integration of new evidence into prior expectations (Blackwood et al., 2001; Moritz and Woodward, 2006; Freeman, 2007) driven by an over-weighting of the prior expectation as compared to incoming sensory evidence (Fletcher and Frith, 2009; Stephan et al., 2009). Such an imbalance between prior expectations and new sensory evidence would result in the discounting of disconfirmatory evidence that runs counter to prior beliefs (Moritz and Woodward, 2006; Woodward et al., 2006, 2007). An imbalance between prior expectations and new sensory evidence could be the result of: atypical sensory processing; atypical processing of prior beliefs; and atypical connectivity between regions associated with sensory processing and prior beliefs. We will discuss each in turn.

\section{SENSORY PROCESSING IN SCHIZOPHRENIA}

A recent study of auditory processing in schizophrenia suggests evidence of dysfunction in the earliest afferent input to the primary auditory cortex, which arrives from subcortical regions, in patients with schizophrenia (Leavitt et al., 2007). Similar findings have been reported with respect to visual processing (e.g., emotional face processing; Gur et al., 2002; Michalopoulou et al., 2008). These findings suggest atypicalities in "bottom-up" sensory processing. However, as can be seen in the ASC literature, supposedly "bottom-up" deficits can be the result of atypical eyegaze patterns or atypical attentional modulation of neural activity. Indeed, individuals with schizophrenia tend to avoid looking at salient regions of the face such as the eyes and mouth and this restricted visual scan path is associated with poorer emotion recognition accuracy (Loughland et al., 2002). Further studies are required to investigate sensory processing, in schizophrenia, in the context of visual scan paths to elucidate whether atypical function of sensory regions can truly be considered a "bottom-up" deficit.

\section{PRIOR BELIEFS IN SCHIZOPHRENIA}

Detecting when something in the environment violates expectations is important, particularly with respect to controlling behaviors such as the direction of eye gaze to sources of new information. In typical control individuals the right PFC has been associated with employing prediction error signals for action selection, which denote the extent to which an incoming sensory stimulus violates expectations (Fletcher et al., 2001; Corlett et al., 2004; Turner et al., 2004; Corlett et al., 2006). In individuals with schizophrenia this region functions atypically (Corlett et al., 2007) and it has been suggested that disruptions in the dopamine and glutamate systems in schizophrenia might result in inaccurate and noisy prediction errors (Corlett et al., 2009; Fletcher and Frith, 2009; Corlett et al., 2011). Although speculative, at present it is possible that these abnormal prediction errors relate to the overly strong effects of priming and expectations discussed above. For instance an inaccurate and noisy representation of the difference between expected and actual events may mean that large violations of expectations are under-weighted and have little influence on learning, and hence abnormally strong priors could prevail (Fletcher and Frith, 2009). To investigate this possibility future studies may employ computational modeling combined with paradigms such as the one we recently employed (Barbalat et al., 2012b) to investigate the role of prediction errors in top-down modulation of social processing. One prediction, based on our previous finding (Barbalat et al., 2012b), is that prediction errors relating to the detection of fearful faces when angry faces are expected would be more noisy for individuals with schizophrenia compared with controls. The noisiness of prediction errors may be hypothesized to be correlated with reaction times such that those individuals with the noisiest prediction errors are the slowest to respond to fearful faces in an anger context.

\section{FUNCTIONAL CONNECTIVITY BETWEEN REGIONS ASSOCIATED WITH SENSORY PROCESSING AND PRIOR BELIEFS IN SCHIZOPHRENIA}

Recent computational models of perception and learning suggest there may be a hierarchy of prediction error driven inferencing devices where lower levels of the hierarchy relate to perception and upper levels are more relevant to beliefs (Friston, 2005; Fletcher and Frith, 2009). Communicating between levels of the hierarchy is important in enabling the updating of behavior according to violations of expectations. Studies using predominately non-social stimuli have led to the suggestion that schizophrenia may be associated with reduced connectivity between brain regions (Friston and Frith, 1995). This theory raises the possibility that schizophrenia is characterized by a lack of communication between different levels of the inferencing hierarchy.

Some researchers have found evidence for this with respect to social stimuli. Ford et al. (2002) investigated event-related electroencephalogram (EEG) coherence whilst participants talked aloud and listened to their own speech while remaining silent. For typical control participants talking, compared to passive listening, elicited greater coherence between frontal and temporal regions, whereas individuals with schizophrenia showed reduced fronto-temporal connectivity during talking (Figure 5).

\section{CONCLUSION}

This paper has discussed evidence that top-down modulation of social perception and imitation is atypical in ASC and schizophrenia. Given the importance of our perception of, and reactions to, others in our daily lives, atypicalities in these abilities may be related to key features of both ASC and schizophrenia.

We have reviewed evidence that both attention and prior expectations modulate social perception in healthy subjects, which might rely on top-down signals from the lateral and the mPFC. In schizophrenia, this top-down modulation of social 


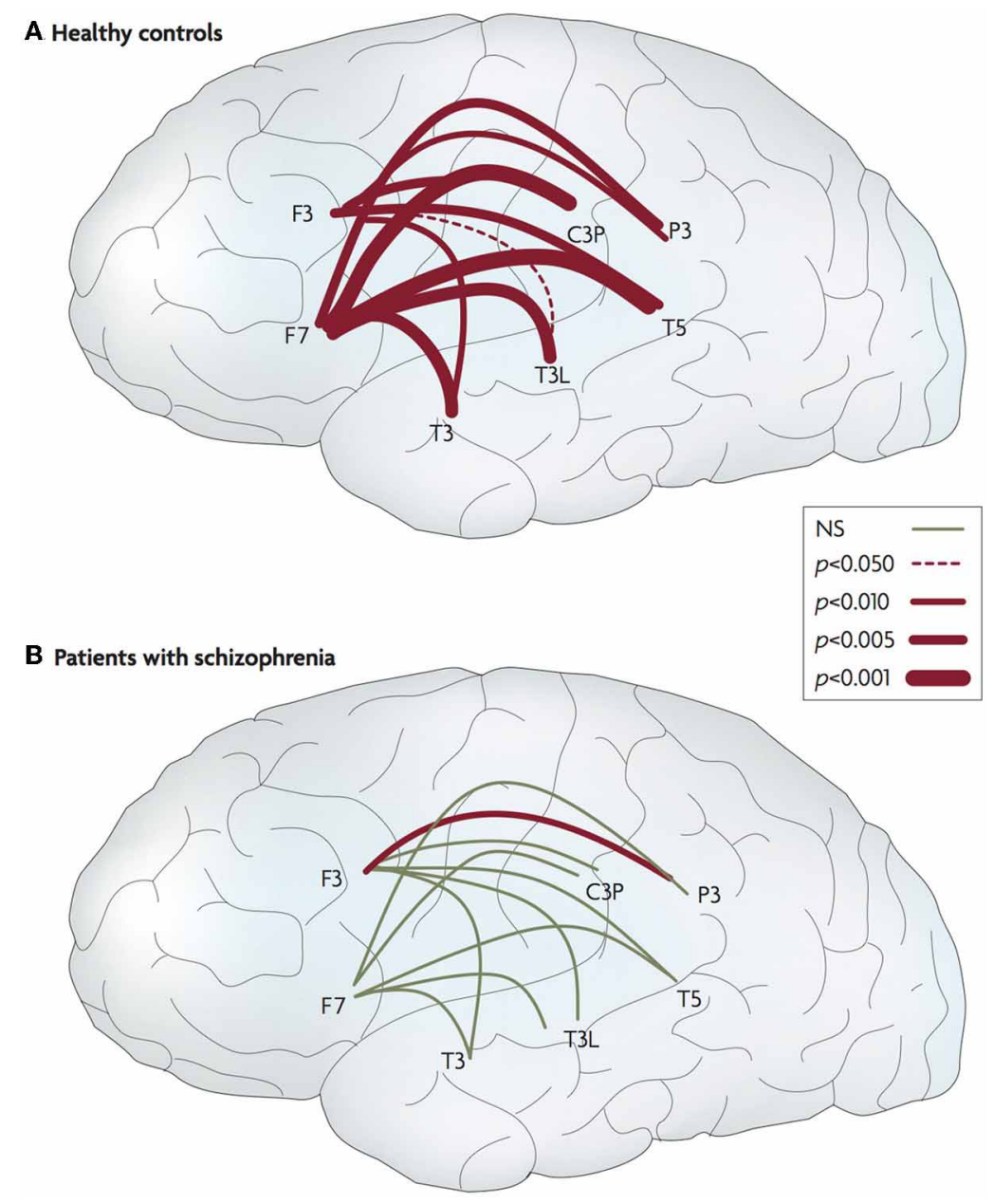

FIGURE 5 | Abnormal connectivity in the brain in schizophrenia.

Lateral views of the left hemisphere of the brain. The red lines connect areas that exhibited greater frontotemporal electroencephalogram coherence during talking than during listening for normal controls and patients with schizophrenia. The thickness of the line indicates the probability level for the $t$-tests that compared the findings. The thicker the line, the larger the difference between the two coherences. In the controls, coherence during talking was greater than during listening for all 20 of the electrode pairs. In the patients, coherences during talking were greater for only two of the pairs (one in each hemisphere). NS, not significant. Data from Ford et al. (2002). Figure and caption is reproduced, with permission from Fletcher and Frith (2009). perception is abnormal in that patients demonstrate atypically strong influences of expectations. For individuals with ASC the opposite appears to be true: where schizophrenia is

\section{REFERENCES}

Aleman, A., Böcker, K. B. E., Hijman, R., de Haan, E. H. F., and Kahn, R. S. (2003). Cognitive basis of hallucinations in schizophrenia: role of top-down information processing. Schizophr. Res. 64, 175-185.

American Psychiatric Association, (1994). Diagnostic and Statistical Manual of Mental Disorders. Washington, DC: American Psychiatric Association.

Andreasen, N. (1983). Scale for the Assessment of Negative Symptoms (SANS). Iowa City, IA: University of Iowa.
Andreasen, N. (1984). Scale for the Assessment of Positive Symptoms (SAPS). Iowa City, IA: University of Iowa.

Avikainen, S., Wohlschläger, S., Liuhanen, S., Hänninen, R., and Hari, R. (2003). Impaired mirror-image imitation in Asperger and high-functioning autistic subjects. Curr. Biol. 13, 339-341.

Bailenson, J., and Yee, N. (2005). Digital chameleons: automatic assimilation of nonverbal gestures in immersive virtual environments. Psychol. Sci. $16,814-819$.

characterized by abnormally strong influences of prior expectations ASC is characterized by abnormally weak top-down modulation.

Barbalat, G., Bazargani, N., and Blakemore, S. J. (2012a). The influence of prior expectations on emotional face perception in adolescence. Cereb. Cortex. doi: 10.1093/cercor/bhs140. [Epub ahead of print].

Barbalat, G., Rouault, M., Bazargani, N., Shergill, S., and Blakemore, S. J. (2012b). The influence of prior expectations on facial expression discrimination in schizophrenia. Psychol. Med. 1-11. [Epub ahead of print].

Bird, G., Catmur, C., Bente, G., Frith, C., and Frith, U. (2006). Attention does not modulate neural responses to social stimuli in autism spectrum disorders. Neuroimage 31, 1614-1624.

Bird, G., Leighton, J., Press, C., and Heyes, C. (2007). Intact automatic imitation of human and robot actions in autism spectrum disorders. Proc. Biol. Sci. 274, 3027-3031. Blackwood, N. J., Howard, R. J., Bentall, R. P., and Murray, R. M. (2001) Cognitive neuropsychiatric models of persecutory delusions. Am. J. Psychiatry 158, 527-539.

Brass, M., Derrfuss, J., and von Cramon, D. Y. (2005). The inhibition of imitative and overlearned 
responses: a functional double dissociation. Neuropsychologia 43, 89-98.

Brass, M., Derrfuss, J., Matthes-von Cramon, G., and von Cramon, D. Y. (2003). Imitative response tendencies in patients with frontal brain lesions. Neuropsychology 17, 265-271.

Brass, M., Zysset, S., and von Cramon, D. Y. (2001). The inhibition of imitative response tendencies. Neuroimage 14, 1416-1423.

Büchel, C., Josephs, O., Rees, G., Turner, R., Frith, C. D., and Friston, K. J. (1998). The functional anatomy of attention to visual motion. A functional MRI study. Brain 121(Pt 7), 1281-1294.

Castelli, F., Frith, C., Happé, F., and Frith, U. (2002). Autism, Asperger syndrome and brain mechanisms for the attribution of mental states to animated shapes. Brain 125, 1839-1849.

Castelli, F., Happé, F., Frith, U., and Frith, C. (2000). Movement and mind: a functional imaging study of perception and interpretation of complex intentional movement patterns. Neuroimage 12, 314-325.

Catmur, C., Walsh, V., and Heyes, C. (2009). Associative sequence learning: the role of experience in the development of imitation and the mirror system. Philos. Trans. R. Soc. Lond. B Biol. Sci. 364, 2369-2380.

Cavada, C., and Goldman-Rakic, P. S. (1989). Posterior parietal cortex in rhesus monkey: II. Evidence for segregated corticocortical networks linking sensory and limbic areas with the frontal lobe. J. Comp. Neurol. 287, 422-445.

Chartrand, T., and Bargh, J. (1999). The chameleon effect: the perceptionbehavior link and social interaction. J. Pers. Soc. Psychol. 76, 893-910.

Chevallier, C., Kohls, G., Troiani, V., Brodkin, E. S., and Schultz, R. T. (2012). The social motivation theory of autism. Trends Cogn. Sci. 16, 231-239.

Cook, J., and Bird, G. (2011a). Atypical social modulation of imitation in autism spectrum conditions. J. Autism Dev. Disord. 42, 1045-1051.

Cook, J., and Bird, G. (2011b). Social attitudes differentially modulate imitation in adolescents and adults. Exp. Brain Res. 211, 601-612.

Corbetta, M., Miezin, F. M., Shulman, G. L., and Petersen, S. E. (1993). A PET study of visuospatial attention. J. Neurosci. 13, 1202-1226.

Corlett, P. R., Aitken, M. R. F., Dickinson, A., Shanks, D. R., Honey, G. D., Honey, R. A. E.,
Robbins, T. W., Bullmore, E. T., and Fletcher, P. C. (2004). Prediction error during retrospective revaluation of causal associations in humans: fMRI evidence in favor of an associative model of learning. Neuron 44, 877-888.

Corlett, P. R., Frith, C. D., and Fletcher, P. C. (2009). From drugs to deprivation: a Bayesian framework for understanding models of psychosis. Psychopharmacology (Berl.) 206, 515-530.

Corlett, P. R., Honey, G. D., Aitken, M. R. F., Dickinson, A., Shanks, D. R., Absalom, A. R., Lee, M., PomarolClotet, E., Murray, G. K., McKenna, P. J., Robbins, T. W., Bullmore, E. T. and Fletcher, P. C. (2006). Frontal responses during learning predict vulnerability to the psychotogenic effects of ketamine: linking cognition, brain activity, and psychosis. Arch. Gen. Psychiatry 63, 611-621.

Corlett, P. R., Honey, G. D., Krystal, J. H., and Fletcher, P. C. (2011). Glutamatergic model psychoses: prediction error, learning, and inference. Neuropsychopharmacology 36, 294-315.

Corlett, P. R., Murray, G. K., Honey, G. D., Aitken, M. R. F., Shanks, D. R., Robbins, T. W., Bullmore, E. T., Dickinson, A., and Fletcher, P. C. (2007). Disrupted predictionerror signal in psychosis: evidence for an associative account of delusions. Brain 130, 2387-2400.

Cornblatt, B. A., and Keilp, J. G. (1994). Impaired attention, genetics, and the pathophysiology of schizophrenia. Schizophr. Bull. 20, 31-46.

Critchley, H. D., Daly, E. M., Bullmore, E. T., Williams, S. C., Van Amelsvoort, T., Robertson, D. M., Rowe, A., Phillips, M., McAlonan, G., Howlin, P., and Murphy, D. G. (2000). The functional neuroanatomy of social behaviour: changes in cerebral blood flow when people with autistic disorder process facial expressions. Brain 123(Pt 11), 2203-2212.

Dapretto, M., Davies, M. S., Pfeifer, J. H., Scott, A. A., Sigman, M., Bookheimer, S. Y., and Iacoboni, M. (2006). Understanding emotions in others: mirror neuron dysfunction in children with autism spectrum disorders. Nat. Neurosci. 9, 28-30.

Daw, N. D., O'Doherty, J. P., Dayan, P., Seymour, B., and Dolan, R. J. (2006). Cortical substrates for exploratory decisions in humans. Nature 441, 876-879.

Dayan, P., Hinton, G. E., Neal, R. M., and Zemel, R. S. (1995). The Helmholtz machine. Neural Comput. 7, 889-904.
Dichter, G. S., and Belger, A. (2008). Atypical modulation of cognitive control by arousal in autism. Psychiatry Res. 164, 185-197.

Dichter, G. S., Bellion, C., Casp, M., and Belger, A. (2010). Impaired modulation of attention and emotion in schizophrenia. Schizophr. Bull. 36, 595-606.

Dosher, B. A., and Lu, Z. L. (1999) Mechanisms of perceptual learning. Vis. Res. 39, 3197-3221.

Fink, G. R., Dolan, R. J., Halligan, P. W., Marshall, J. C., and Frith, C. D. (1997). Space-base and objectbased visual attention: shared and specific neural domains. Brain 120, 2013-2028.

Fletcher, P. C., Anderson, J. M., Shanks, D. R., Honey, R., Carpenter, T. A., Donovan, T., Papadakis, N., and Bullmore, E. T. (2001). Responses of human frontal cortex to surprising events are predicted by formal associative learning theory. Nat. Neurosci. 4, 1043-1048.

Fletcher, P. C., and Frith, C. D. (2009). Perceiving is believing: a Bayesian approach to explaining the positive symptoms of schizophrenia. Nat. Rev. Neurosci. 10, 48-58.

Ford, J. M., Mathalon, D. H., Whitfield, S., Faustman, W. O., and Roth, W. T. (2002). Reduced communication between frontal and temporal lobes during talking in schizophrenia. Biol. Psychiatry 51, 485-492.

Freeman, D. (2007). Suspicious minds: the psychology of persecutory delusions. Clin. Psychol. Rev. 27, 425-457.

Friston, K. (2005). A theory of cortical responses. Philos. Trans. R. Soc. Lond. B Biol. Sci. 360, 815-836.

Friston, K. J., and Frith, C. D. (1995). Schizophrenia: a disconnection syndrome? Clin. Neurosci. 3, 89-97.

Frith, C. D. (2003). What do imaging studies tell us about the neural basis of autism? Novartis Found. Symp. 251, 149-66.

Gur, R. E., McGrath, C., Chan, R. M., Schroeder, L., Turner, T., Turetsky, B. I., Kohler, C., Alsop, D., Maldjian, J., Ragland, J. D., and Gur, R. C. (2002). An fMRI study of facial emotion processing in patients with schizophrenia. Am. J. Psychiatry 159, 1992-1999.

Haddock, G., McCarron, J., Tarrier, N. and Faragher, E. B. (1999). Scales to measure dimensions of hallucinations and delusions: the psychotic symptom rating scales (PSYRATS). Psychol. Med. 29, 879-889.

Hadjikhani, N., Joseph, R. M., Snyder J., Chabris, C. F., Clark, J., Steele, S., McGrath, L., Vangel, M., Aharon, I.,
Feczko, E., Harris, G. J., and TagerFlusberg, H. (2004). Activation of the fusiform gyrus when individuals with autism spectrum disorder view faces. Neuroimage 22, 1141-1150.

Hampton, A. N., Bossaerts, P., and O'Doherty, J. P. (2006). The role of the ventromedial prefrontal cortex in abstract state-based inference during decision making in humans. J. Neurosci. 26, 8360-8367.

Happé, F., Brownell, H., and Winner, E. (1999). Acquired "theory of mind" impairments following stroke. Cognition 70, 211-240.

Heiser, M., Iacoboni, M., Maeda, F., Marcus, J., and Mazziotta, J. C. (2003). The essential role of Broca's area in imitation. Eur. J. Neurosci. 17, 1123-1128.

Hooker, C. I., Tully, L. M., Verosky, S. C., Fisher, M., Holland, C., and Vinogradov, S. (2011). Can I trust you? Negative affective priming influences social judgments in schizophrenia. J. Abnorm. Psychol. 120, 98-107.

Höschel, K., and Irle, E. (2001). Emotional priming of facial affect identification in schizophrenia. Schizophr. Bull. 27, 317-327.

Iacoboni, M. (2005). Neural mechanisms of imitation. Curr. Opin. Neurobiol. 15, 632-637.

Iacoboni, M. (2009). Imitation, empathy, and mirror neurons. Annu. Rev. Psychol. 60, 653-670.

Iacoboni, M., Woods, R., Brass, M., Bekkering, H., Mazziotta, J., and Rizzolatti, G. (1999). Cortical mechanisms of human imitation. Science 286, 2526-2528.

Ilankovic, L. M., Allen, P. P., Engel, R., Kambeitz, J., Riedel, M., Müller, N., and Hennig-Fast, K. (2011). Attentional modulation of external speech attribution in patients with hallucinations and delusions. Neuropsychologia 49, 805-812.

Kana, R. K., Keller, T. A., Cherkassky, V. L., Minshew, N. J., and Just, M. A. (2009). Atypical frontal-posterior synchronization of theory of mind regions in autism during mental state attribution. Soc. Neurosci. 4, 135-152.

Kastner, S., and Ungerleider, L. G. (2001). The neural basis of biased competition in human visual cortex. Neuropsychologia 39, 1263-1276.

Kiehl, K. A., and Liddle, P. F. (2001). An event-related functional magnetic resonance imaging study of an auditory oddball task in schizophrenia. Schizophr. Res. 48, 159-171.

Kirino, E., Belger, A., Goldman-Rakic, P., and McCarthy, G. (2000). Prefrontal activation evoked by infrequent target and novel stimuli 
in a visual target detection task: an event-related functional magnetic resonance imaging study. J. Neurosci. 20, 6612-6618.

Kraskov, A., Dancause, N., Quallo, M. M., Shepherd, S., and Lemon, R. N. (2009). Corticospinal neurons in macaque ventral premotor cortex with mirror properties: a potential mechanism for action suppression? Neuron 64, 922-930.

Lakin, J., and Chartrand, T. (2003). Using nonconscious behavioral mimicry to create affiliation and rapport. Psychol. Sci. 14, 334-339.

Leavitt, V. M., Molholm, S., Ritter, W., Shpaner, M., and Foxe, J. J. (2007). Auditory processing in schizophrenia during the middle latency period (10-50 ms): highdensity electrical mapping and source analysis reveal subcortical antecedents to early cortical deficits. J. Psychiatry Neurosci. 32, 339-353.

Leighton, J., Bird, G., Orsini, C., and Heyes, C. (2010). Social attitudes modulate automatic imitation. J. Exp. Soc. Psychol. 46, 905-910.

Lhermitte, F. (1986). Human autonomy and the frontal lobes. Part II: patient behavior in complex and social situations: the "environmental dependency syndrome." Ann. Neurol. 19, 335-343.

Lord, C., Rutter, M., Goode, S., Heemsbergen, J., Jordan, H., Mawhood, L., and Schopler, E. (1989). Autism diagnostic observation schedule: a standardized observation of communicative and social behavior. J. Autism Dev. Disord. 19, 185-212.

Loth, E., Gómez, J. C., and Happé, F. (2010). When seeing depends on knowing: adults with autism spectrum conditions show diminished top-down processes in the visual perception of degraded faces but not degraded objects. Neuropsychologia 48, 1227-1236.

Loughland, C. M., Williams, L. M., and Gordon, E. (2002). Visual scanpaths to positive and negative facial emotions in an outpatient schizophrenia sample. Schizophr. Res. 55, 159-170.

McIntosh, D., Reichmann-Decker, A., Winkielman, P., and Wilbarger, J. (2006). When the social mirror breaks: deficits in automatic, but not voluntary, mimicry of emotional facial expressions in autism. Dev. Sci. 9, 295-302.

Michalopoulou, P. G., Surguladze, S., Morley, L. A., Giampietro, V. P., Murray, R. M., and Shergill, S. S. (2008). Facial fear processing and psychotic symptoms in schizophrenia: functional magnetic resonance imaging study. Br. J. Psychiatry 192, 191-196.

Morey, R. A., Inan, S., Mitchell, T. V., Perkins, D. O., Lieberman, J. A., and Belger, A. (2005). Imaging frontostriatal function in ultra-high-risk, early, and chronic schizophrenia during executive processing. Arch. Gen. Psychiatry 62, 254-262.

Moritz, S., and Woodward, T. S. (2006). A generalized bias against disconfirmatory evidence in schizophrenia. Psychiatry Res. 142, 157-165.

Oberman, L., Hubbard, E., McCleery, J., Altschuler, E., Ramachandran, V., and Pineda, J. (2005). EEG evidence for mirror neuron dysfunction in autism spectrum disorders. Brain Res. Cogn. Brain Res. 24, 190-198.

di Pellegrino, G., Fadiga, L., Fogassi, L., Gallese, V., and Rizzolatti, G. (1992). Understanding motor events: a neurophysiological study. Exp. Brain Res. 91, 176-180.

Penn, D. L., Sanna, L. J., and Roberts, D. L. (2008). Social cognition in schizophrenia: an overview. Schizophr. Bull. 34, 408-411.

Penn, D. L., Spaulding, W., Reed, D., Sullivan, M., Mueser, K. T., and Hope, D. A. (1997). Cognition and social functioning in schizophrenia. Psychiatry 60, 281-291.

Pessoa, L., and Adolphs, R. (2010). Emotion processing and the amygdala: from a "low road" to "many roads" of evaluating biological significance. Nat. Rev. Neurosci. 11, 773-783.

Pessoa, L., Kastner, S., and Ungerleider, L. G. (2002). Attentional control of the processing of neural and emotional stimuli. Brain Res. Cogn. Brain Res. 15, 31-45.

Pierce, K., Müller, R. A., Ambrose, J., Allen, G., and Courchesne, E. (2001). Face processing occurs outside the fusiform "face area" in autism: evidence from functional MRI. Brain 124, 2059-2073.

Press, C., Richardson, D., and Bird, G. (2010). Intact imitation of emotional facial actions in autism spectrum conditions. Neuropsychologia 48, 3291-3297.

Rogers, S., Hepburn, S., Stackhouse, T., and Wehner, E. (2003). Imitation performance in toddlers with autism and those with other developmental disorders. J. Child Psychol. Psychiatry 44, 763-781.

Schultz, R. T., Gauthier, I., Klin, A., Fulbright, R. K., Anderson, A. W. Volkmar, F., Skudlarski, P., Lacadie, C., Cohen, D. J., and Gore, J. C. (2000). Abnormal ventral temporal cortical activity during face discrimination among individuals with autism and Asperger syndrome. Arch. Gen. Psychiatry 57, 331-340.

Shih, P., Shen, M., Ottl, B., Keehn, B., Gaffrey, M., and Müller, R. (2010). Atypical network connectivity for imitation in autism spectrum disorder. Neuropsychologia 48, 2931-2939.

Spengler, S., Bird, G., and Brass, M. (2010a). Hyperimitation of actions is related to reduced understanding of others' minds in autism spectrum conditions. Biol. Psychiatry 68 , 1148-1155.

Spengler, S., von Cramon, D. Y., and Brass, M. (2009). Control of shared representations relies on key processes involved in mental state attribution. Hum. Brain Mapp. 30, 3704-3718.

Spengler, S., von Cramon, D. Y., and Brass, M. (2010b). Resisting motor mimicry: control of imitation involves processes central to social cognition in patients with frontal and temporo-parietal lesions. Soc. Neurosci. 5, 401-416.

Stephan, K. E., Friston, K. J., and Frith, C. D. (2009). Dysconnection in schizophrenia: from abnormal synaptic plasticity to failures of self-monitoring. Schizophr. Bull. 35 , 509-527.

Summerfield, C., and Egner, T. (2009). Expectation (and attention) in visual cognition. Trends Cogn. Sci. 13, 403-409.

Summerfield, C., Egner, T., Greene, M., Koechlin, E., Mangels, J., and Hirsch, J. (2006). Predictive codes for forthcoming perception in the frontal cortex. Science 314, 1311-1314.

Summerfield, C., and Koechlin, E. (2008). A neural representation of prior information during perceptual inference. Neuron 59, 336-347.

Suslow, T., Roestel, C., and Arolt, V. (2003). Affective priming in schizophrenia with and without affective negative symptoms. Eur. Arch. Psychiatry Clin. Neurosci. 253 292-300.

Turner, D. C., Aitken, M. R. F., Shanks D. R., Sahakian, B. J., Robbins, T. W. Schwarzbauer, C., and Fletcher, P. C. (2004). The role of the lateral frontal cortex in causal associative learning: exploring preventative and superlearning. Cereb. Cortex 14, 872-880.

Ungerleider, L. G., Gaffan, D., and Pelak, V. S. (1989). Projections from inferior temporal cortex to prefrontal cortex via the uncinate fascicle in rhesus monkeys. Exp. Brain Res. 76, 473-484.

van Baaren, R., Holland, R., Kawakami, K., and van Knippenberg, A. (2004).
Mimicry and prosocial behavior. Psychol. Sci. 15, 71-74.

Vuilleumier, P., Armony, J. L., Driver, J., and Dolan, R. J. (2001). Effects of attention and emotion on face processing in the human brain: an event-related fMRI study. Neuron 30, 829-841.

Wang, Y., Newport, R., and de C Hamilton, A. F. (2011a). Eye contact enhances mimicry of intransitive hand movements. Biol. Lett. 7, 7-10.

Wang, Y., Ramsey, R., and de C Hamilton, A. F. (2011b). The control of mimicry by eye contact is mediated by medial prefrontal cortex. J. Neurosci. 31, 12001-12010.

Woodward, T. S., Buchy, L., Moritz, S., and Liotti, M. (2007). A bias against disconfirmatory evidence is associated with delusion proneness in a nonclinical sample. Schizophr. Bull. 33, 1023-1028.

Woodward, T. S., Moritz, S., Cuttler, C., and Whitman, J. C. (2006). The contribution of a cognitive bias against disconfirmatory evidence (BADE) to delusions in schizophrenia. J. Clin. Exp. Neuropsychol. 28, 605-617.

Woodward, T. S., Moritz, S., Menon, M., and Klinge, R. (2008). Belief inflexibility in schizophrenia. Cogn. Neuropsychiatry 13, 267-277.

Wyart, V., Nobre, A. C., and Summerfield, C. (2012). Dissociable prior influences of signal probability and relevance on visual contrast sensitivity. Proc. Natl. Acad. Sci. U.S.A. 109, 3593-3598.

Conflict of Interest Statement: The authors declare that the research was conducted in the absence of any commercial or financial relationships that could be construed as a potential conflict of interest.

Received: 29 February 2012; accepted: 29 May 2012; published online: 15 June 2012

Citation: Cook J, Barbalat $G$ and Blakemore $S$ (2012) Top-down modulation of the perception of other people in schizophrenia and autism. Front. Hum. Neurosci. 6:175. doi: 10.3389/ fnhum.2012.00175

Copyright (c) 2012 Cook, Barbalat and Blakemore. This is an open-access article distributed under the terms of the Creative Commons Attribution Non Commercial License, which permits noncommercial use, distribution, and reproduction in other forums, provided the original authors and source are credited. 